BULL. AUSTRAL. MATH. SOC.

$58 A 99,53 \mathrm{C} 05$

VOL. $10(1974), 71-77$.

\title{
Recurrent tensors and holonomy group
}

\section{D.K. Datta}

Let $M$ be a connected $C^{\infty}$ manifold of dimension $n$ with a linear connection $\Gamma$. A method is being introduced here to study the action of the holonomy group and the restricted holonomy group of $\Gamma$ on a recurrent tensor. The main result of this paper is that if the recurrence covector $W$ of a recurrent tenscr $S$ on $M$ is an exact form then the tensor $S$ is invariant under the holonomy group of $\Gamma$ and if $W$ is a closed form then $S$ is invariant under the restricted holonomy group of $\Gamma$. In the last section, this result is applied to some particular cases including the case of a riemannian manifold with recurrent curvature.

\section{Introduction}

Let $M$ be a connected $C^{\infty}$ manifold of dimension $n$ and let a linear connection $\Gamma$ be given on $M$. A tensor field $S$ of type $(r, s)$ on $M$ is said to be recurrent if [4],

$$
\nabla S=W \otimes S,
$$

where $W$ is a differential $l$-form on $M$ and $\nabla$ denotes covariant derivative with respect to $\Gamma$. The 1 -form $W$ is called the recurrence covector.

The purpose of this paper is to find the conditions under which a

Received 17 September 1973. This paper was written while the author was visiting the Australian National University as an Honorary Fellow. He is grateful to the Department of Mathematics for its hospitality. 
recurrent tensor will be invariant by the action of the holonomy group or the restricted holonom group of $\Gamma$.

\section{Preliminaries}

For a point $p$ of $M$ let $\left(x_{1}, \ldots, x_{n}\right)$ be a local coordinate system in a coordinate neighbourhood $U$ of $p$. Let $s_{j_{1} \ldots j_{s}}^{i_{1} \ldots i_{r}}$ and $w_{i}$ respectively be the components of $S$ and $W$ with respect to the coordinate system on $U$. Then the condition for $S$ to be recurrent can be written as

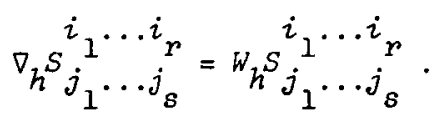

For the 1 -form $W$, we have the relation

$$
d W=\operatorname{Alt}(\nabla W),
$$

where $d W$ denotes the exterior derivative of $W$ and $\operatorname{Alt}(\nabla W)$ is given locally by $\operatorname{Alt}(\nabla W)=\nabla_{j} W_{i}-\nabla_{i} W_{j}$. This shows that $W$ is a closed form if and only if $\nabla W$ is symmetric.

If $W$ is an exact form we have the following

THEOREM 1.1. Let $S$ be a recurrent tensor fiezd on a connected $c^{\infty}$ manifold $M$ such that the recurrence covector is an exact form. Then there exists a function $\sigma$ on $M$ such that $\sigma S$ is a parallel tensor field on $s$.

Proof. Let $\nabla=W \otimes S$. If $W$ is an exact form then there exists a function $f$ on $M$ such that $W=d f$. Taking $\sigma=e^{-f}$, it follows that $\nabla\left(e^{-f_{S}}\right)=0$.

\section{Main theorem}

Let $p$ be a point in $M$ and let $\tau: t \rightarrow x(t),(0 \leq t \leq 1)$, be a loop at $p$, that is, $\tau$ is a sectionally smooth closed curve with $\tau(0)=\tau(1)=p$. Since any sectionally smooth curve in a smooth manifold may be regarded as one each of whose smooth sections lies in a coordinate 
neighbourhood, it will be sufficient for our purpose to assume that $\tau$ is contained in a single coordinate neighbourhood $U$ of $p$. Let $S$ be a recurrent tensor with recurrence covector $W$. We shall denote by $S_{x(t)}$ the assignment of the tensor field $S$ at $x(t)=\left(x_{1}(t), \ldots, x_{n}(t)\right)$. Then along $\tau$, we get

$$
\begin{aligned}
\nabla_{h} S_{x(t)} \frac{d x h}{d t} & =w_{h}(x(t)) S_{x(t)} \frac{d x_{h}}{d t} \\
& =f(t) S_{x(t)},
\end{aligned}
$$

where $f(t)=W_{h}(x(t)) \frac{d x_{h}}{d t}$.

To determine the action of the holonomy group of $\Gamma$ on the recurrent tensor $S$, we construct a tensor field $A$ of the same type as $S$ and defined along $\tau$ such that

$$
A_{x(0)}=S_{x(0)}=S_{p}
$$

and

$$
\nabla_{h} A x(t) \frac{d x_{h}}{d t}=0
$$

The functions $A_{x}(t)$ defined along $\tau$ by

$$
A_{x(t)}=\exp \left(-\int_{0}^{t} f(t) d t\right) S_{x(t)}
$$

will give such a tensor field along $\tau$ since $A_{x(0)}=S_{x(0)}$ and

$$
\begin{aligned}
\nabla_{h} A_{x}(t) \frac{d x_{h}}{d t} & =\nabla_{h}\left[\exp \left(-\int_{0}^{t} f(t) d t\right) S_{x(t)}\right] \frac{d x_{h}}{d t} \\
& =\exp \left(-\int_{0}^{t} f(t) d t\right)\left(\nabla_{h} S_{x(t)}\right) \frac{d x_{h}}{d t}+\nabla_{h}\left(\exp \left(-\int_{0}^{t} f(t) d t\right)\right) S_{x(t)} \frac{d x_{t}}{d t} \\
& =\exp \left(-\int_{0}^{t} f(t) d t\right) f(t) S_{x(t)}-\exp \left(-\int_{0}^{t} f(t) d t\right) f(t) S_{x(t)} \\
& =0 .
\end{aligned}
$$

Therefore, if we denote by $\tau$ the element in the holonomy group of $\Gamma$ 
with reference point $p$ corresponding to the curve $\tau: t \rightarrow x(t)$ then

$$
\begin{aligned}
\tau\left(S_{p}\right) & =\tau\left(A_{p}\right) \\
& =\exp \left(-\int_{0}^{1} f(t) d t\right) S_{x(1)} \\
& =\rho S_{p},
\end{aligned}
$$

where $\rho=\exp \left(-\int_{0}^{1} f(t) d t\right)=\exp \left(-\int_{\tau} f(t) d t\right)$.

$\rho$ will be different for different elements of the holonomy group of $\Gamma$ with reference point $p$. The recurrent tensor $S$ will be invariant under $\tau$ if $p=1$.

From the theory of integration of forms, it follows that $\rho=\exp \left(-\int_{\tau} f(t) d t\right)=\exp \left(-\int_{\tau} W\right)=1$, that is, $\int_{\tau} W=0$ if

(i) $W$ is a closed form and $\tau$ is homotopic to zero, that is, $\tau$ is an element of the restricted holonomy group of $\Gamma$, or if,

(ii) $W$ is an exact form.

Since the holonomy group of $\Gamma$ with different reference points of a connected $C^{\infty}$ manifold are isomorphic, we have proved the following:

THEOREM 2.1. Let $M$ be a connected $c^{\infty}$ manifold with a linear connection $\Gamma$ and let $S$ be a recurrent tensor on $M$. If the recurrence covector $W$ of $S$ is a closed form then $S$ is invariant under the restricted holonomy group of $\Gamma$ and if $W$ is an exact form then $S$ is invariant under the holonomy group of $\Gamma$.

\section{Applications}

In this section we discuss applications of Theorem 2.1 to some interesting particular cases. For example, if $S$ is a vector field on $M$ then we get

THEOREM 3.1. Let $M$ be a connected $C^{\infty}$-manifold with a linear connection $\Gamma$ and let $S$ be a vector field on $M$. Then the holonomy 
Group of $\Gamma$ has an invariant 1-plane.

Proof. This follows from the relation $\tau\left(S_{p}\right)=\rho S_{p}$ as shown in 52 . Clearly, the 1-plane generated by $S$ is invariant under the holonomy group of $\Gamma$.

In [2] Sasaki and Goto have proved certain theorems when the restricted holonomy group of a complete riemannian connection fixes $r$ vectors and these $r$ vectors span an $r$-dimensional plane invariant under the holonomy group of the connection. In the following theorem we give an example of a set of vectors which will satisfy these conditions with respect to a linear connection on a connected $C^{\infty}$ manifold J

THEOREM 3.2. Let $M$ be a connected $C^{\infty}$ manifold with a linear connection $\Gamma$ and let $\left\{v_{A} \mid A=1, \ldots, r\right\}$ be a set of yector fiezds on $M$ such that $V_{1}, \ldots, V_{r}$ are linearly independent at least at one point of $M$ and $\nabla V_{A}=W \otimes V_{A} \quad(A=1, \ldots, r)$ for some closed form $W$. Then

(a) $V_{1}, \ldots, V_{r}$ are invariant under the restricted holonomy group of $\Gamma$,

(b) $V_{1}, \ldots, V_{r}$ are linearly independent at every point of $M$ and

(c) span of $\left\{V_{1}, \ldots, V_{p}\right\}$ is invariant under the holonomy group of $\Gamma$.

Proof. Part $(a)$ follows from Theorem 2.1 since each of $v_{1}, \ldots, v_{r}$ is a recurrent tensor with a closed form as recurrence covector.

Part (b) follows from Corollary 2.3 of Ludden [1] since $v_{1}, \ldots, v_{r}$ form a set of perfect tensors.

Part (c) follows from Theorem 3.1.

For a riemannian connection with $\nabla R=W \otimes R$, where $R$ is the curvature tensor, it is known that $\nabla W$ is symetric so that $W$ is a closed form [3, 4]. Therefore, we get

THEOREM 3.3. FOr a Miemannian manifold with recurrent curvature, the curvature tensor is invariant under the restricted holonomy group of the 
riemannian connection.

For a riemannian manifold with recurrent curvature, it can be shown that other tensors obtained from the curvature tensor are also recurrent with the same recurrence covector. According to Theorem 2.1 and the fact that $\nabla W$ is symmetric, it follows that all such tensors are also invariant under the restricted holonomy group of the riemannian connection.

Wong [5] has considered various types of recurrent tensors which satisfy either the condition that the recurrence covector is a closed form or the condition that the recurrence covector is an exact form. For example, if a recurrent tensor of type $(r, r)$ is not almost parallel (a recurrent tensor $S$ on $M$ is almost parallel if there exists on $M$ some linear connection with respect to which $S$ is parallel) then the recurrerce covector is a closed form. Therefore, we get

THEOREM 3.4. Let $S$ be a recurrent tensor of type $(r, r)$ on a connected $C^{\infty}$ manifold with a linear connection $\Gamma$. If $S$ is not almost paralzel then $S$ is invariant under the restricted holonomy group of $\Gamma$.

Also, if a recurrent tensor of type $(r, r)$ on $M$ is not completely nilpotent [5] then the recurrence covector is globally a gradient, that is, the recurrence covector is an exact form. Thus:

THEOREM 3.5. Let $S$ be a recurrent tensor of type $(r, r)$ on a connected $C^{\infty}$ manifold $M$ with a linear connection $\Gamma$. If $S$ is not completely nilpotent then $S$ is invariant under the holonomy group of $\Gamma$.

The main theorem of this paper may also be generalized to the case of a set of perfect tensors [1].

\section{References}

[1] Gerald D. Ludden, "Perfect tensors on a manifold", J. Differential Geometry 2 (1968), 41-53.

[2] Shigeo Sasaki and Morikuni Goto, "Some theorems on holonomy groups of Riemannian manifolds", Trans. Amer. Math. Soc. 80 (1955), 148-158. 
[3] A.G. Walker, "On Ruse's spaces of recurrent curvature", Proc. London Math. Soc. (2) 52 (1951), 36-64.

[4] Yung-Chow Wong, "Recurrent tensors on a linearly connected differentiable manifold", Trans. Amer. Math. Soc. 99 (1961), $325-341$.

[5] Yung-Chow Wong, "Existence of linear connections with respect to which given tensor fields are parallel or recurrent", Nagoya Math. J. 24 (1964), 67-108.

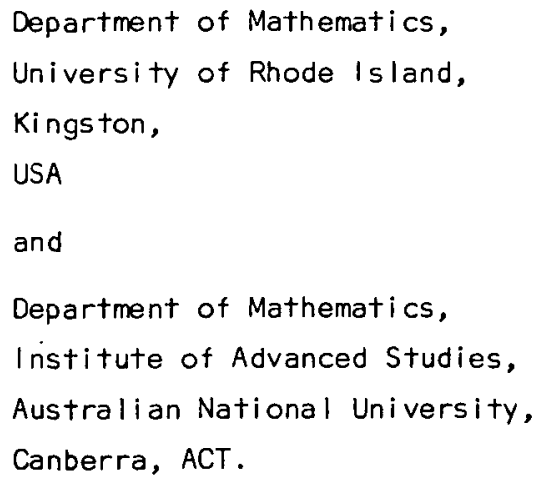

